\title{
Yoga in Pregnancy
}

\author{
M Aruna Devi ${ }^{1}$, Kandasamy Renuka ${ }^{2}$
}

\begin{abstract}
Yoga is originated in India considered as an ancient mind-body practice and nowadays it has been recognized and practiced to promote and to maintain health in these conditions: immunological, neuromuscular, psychological, and pain and determines the mind-body practice which encompasses the conventional substantial pose and can integrate alternative factors, such as meditation and breathing activity. Yoga helps in the regulation of the nervous system, physiological functioning, and improves psychological well-being and physical fitness.

Keywords: Attention-deficit/hyperactivity disorder, Pregnancy, Yoga.

Pondicherry Journal of Nursing (2020): 10.5005/jp-journals-10084-12158
\end{abstract}

\section{INTRODUCTION}

Pregnancy is a state in which the mother goes through various changes in physiological and psychological. There is a demand to manage the results of the changes during all the stages of pregnancy and labor with physical, emotional, and mental. ${ }^{1}$ Risk factors, such as anxiety and stress related to maternal, are considered for potential negative consequences for fetus life. ${ }^{2}$ The attention-deficit/hyperactivity disorder (ADHD) or decreased performance in executive function aspects. ${ }^{3-6}$ Hence, it is essential to balance the stress related to maternal and supply expectant mom with enduring actions for the unavoidable tensions aids in improving the soul and to expand the well-being of infant.

\section{Substantial Changes during Pregnancy}

There are many changes that occur during pregnancy, which are reversible after delivery with proper care.

- Fatigue and exhaustion due to an increase in oxygen consumption.

- Cardiovascular changes, such as increased blood pressure, heart rate, and pedal edema.

- Muscular and ligamentous laxity due to hormonal changes and fetal growth.

- Postural imbalances will occur due to the altered center of gravity.

- Postural disorders, such as exaggerated lordosis, due to sagging shoulders (breast enlargement and fetal growth). ${ }^{7}$

\section{Emotional Changes during Pregnancy}

- The mother will feel of insecurity and nervousness.

- Depression will occur in physiological changes of pregnancy.

- Assurance and motivation are needed.

- Loss of appetite. ${ }^{7}$

\section{Yoga Poses for Women during Pregnancy}

Exercise during pregnancy is essential. The following pregnancy yoga poses will help the pregnant mother during pregnancy: ${ }^{8}$
${ }^{1}$ Department of Community Health Nursing, Kasturba Gandhi Nursing College, Puducherry, India

${ }^{2}$ Department of Medical Surgical Nursing, Kasturba Gandhi Nursing College, Puducherry, India

Corresponding Author: M Aruna Devi, Department of Community Health Nursing, Kasturba Gandhi Nursing College, Puducherry, India, Phone: +91 8870713858, e-mail: arunatanushka@gmail.com

How to cite this article: Aruna Devi M, Renuka K. Yoga in Pregnancy. Pon J Nurs 2020;13(4):96-100.

Source of support: Nil

Conflict of interest: None

\section{Marjariasana (Cat Stretch)}

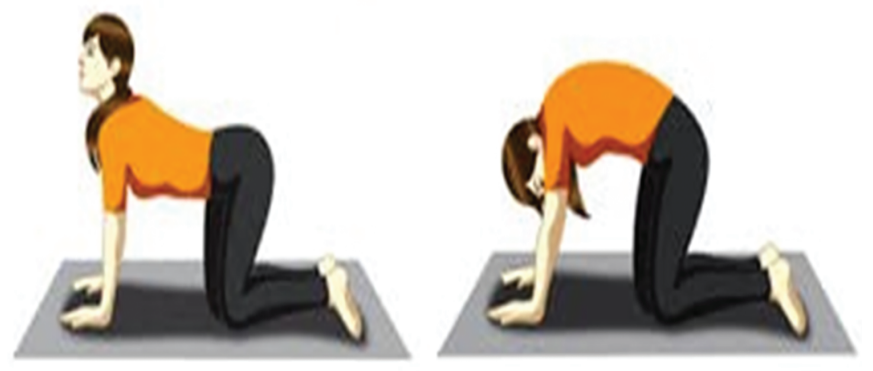

\section{Steps}

- Come onto your palms and knees, with your palms shoulderwidth apart below your shoulders and your knees hip-width apart below your hips.

- Breathe in and raise the head and gently bend the spine close to the floor.

- While breathe out, bring your chin toward your chest and gently arch the spine upward.

- Repeat for 5 to 10 times.

- Do not strain yourself.

\section{Advantages}

- It helps to ease the stiffness in the neck and shoulders.

- Have the spine elastic in nature. As the pregnancy advances the back needs to bear more weight. ${ }^{9}$ 
- Tones the abdominal muscles.

- Advances the blood circulation and assures the private parts are well cherished.

\section{Konasana-I (Standing Sideways Bending One Arm)}
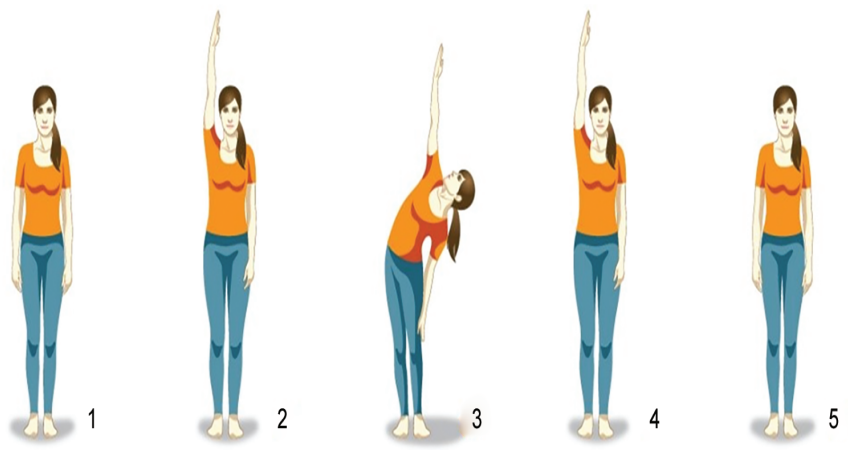

Steps

- Breathe in, and raise your left arm. During breathe out, bend to the right, and see your left palm. Expire the air out, bring the left arm down.

- Repeat on the other side.

\section{Advantages}

- Have the spine extensible.

- Aids in preventing constipation, a usual symptom of pregnancy.

\section{Konasana-II (Standing Sideways Using Both Arms)}

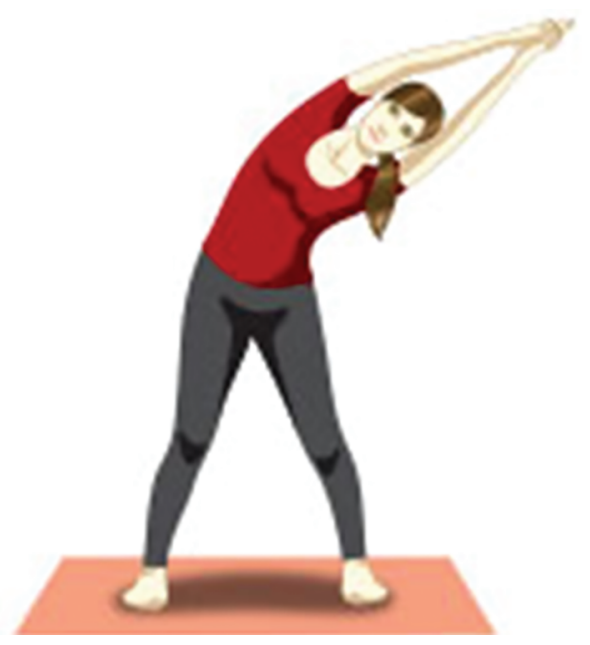

Steps

- Inhale the air, lift the arms above the head and interlace the palms, interlacing your fingers to form a steeple position.

- Breathing out, bend to the right. Hold. Breathing in, return to the standing position. Exhaling the air, bring your arms down. Repeat on the other side.

\section{Advantages}

- Extend and tone the upper and lower extremities and abdominal organs.

- Stretches and exercises the spine.

\section{Virabhadrasana (Warrior Pose)}

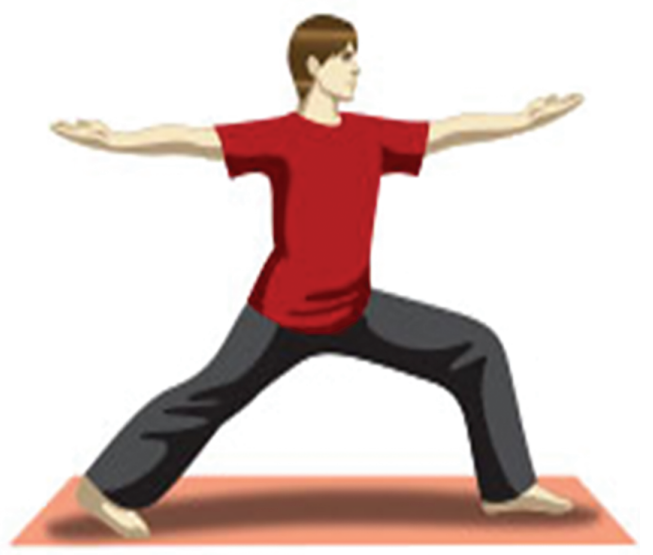

Steps

- Pose with your feet 3-4 feet apart. Twist the right foot out by $90^{\circ}$, and the left foot in by $15^{\circ}$.

- Breathing in, raise your upper extremity at the level of the shoulder.

- Exhale, and then flex your right knee, keeping it in line with the ankle, and gently working to bring your right thigh parallel to the floor.

- Twist the head toward the right side and look across the hand in the right.

\section{Advantages}

- Aids to balance the body.

- Builds up the arms, legs, and lower back.

- Increases energy levels within the body.

\section{Trikonasana (Triangle Pose)}

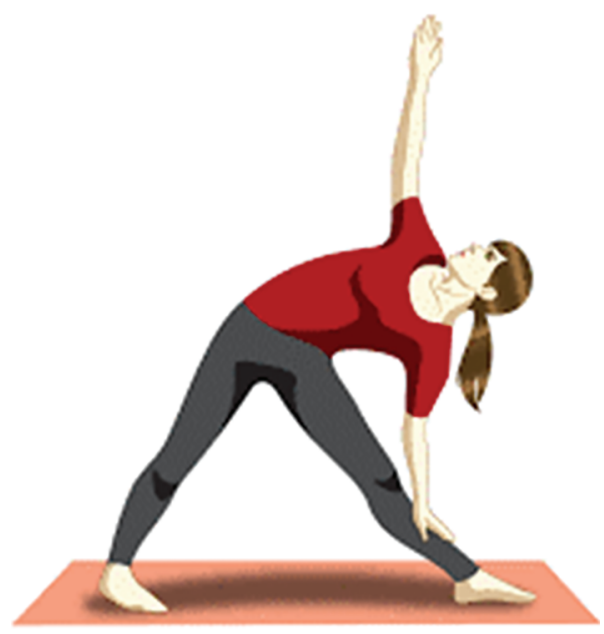

Steps

- Stand with your feet 2-3 feet apart facing forward.

- Turn the head, left side foot to the left side and bring your left side hand down to the left side foot.

- Place the palm to the left side hand on the left side foot and keep your right hand upward. Breathing in, and bring your arms lateral to the floor. 
- Exhale, extend, and flex on your right side to bring the right hand closer to the right foot.

- Hold breathing in, slowly come up, and then relax.

\section{Advantages}

- Maintains a balance between physical and mental health. Exclusively for expectant mom since their center of gravity shifts.

- Extends and widens the hips which aid in delivery.

- Minimizes low back pain and maternal stress.

\section{Badhakonasana (Butterfly Pose)}

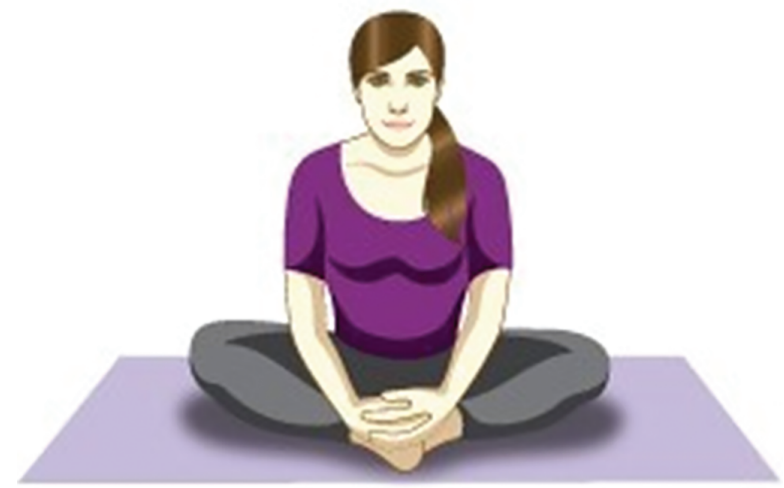

Steps

- Pose with your legs as shown in the image. Turn your knees and put the soles of the feet altogether, holding the heels as close to the body as possible.

- Loosen your inner thighs.

- Grip your feet with two hands.

- Smoothly recoil the knees up and down, utilizing the elbows as levers to press the lower extremities down. Do not use any force. Repeat up to 20 to 30 times. Straighten your legs and calm down.

\section{Advantages}

- Promote flexibility in the hip and groin region.

- Extends the thighs and knees, minimizing pain.

- Reduces fatigue.

- Aids in facilitating smooth delivery.

\section{Viparita Karani (Legs Up the Wall Pose)}

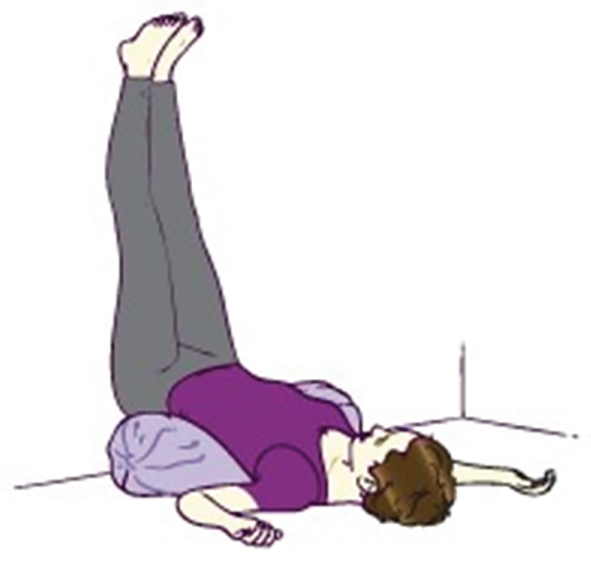

Steps

- Lift both legs up toward the sky, breathe in.

- Do not bend the knees if possible.

- Breathe out, lower the legs to the ground.

- Repeat this for two or three times.

\section{Advantages}

- Alleviate backache.

- Advances the blood circulation to the pelvic region.

- Minimizes edematous ankles and varicose veins-a usual symptom of pregnancy.

\section{Savasana (Corpse Pose)}

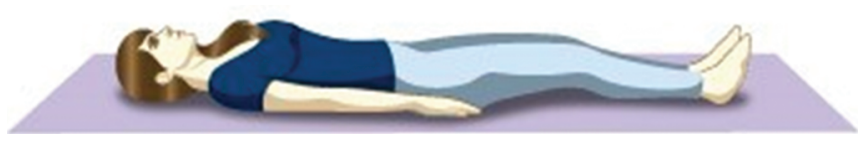

Steps

- Lie in supine position on with closed eyes.

- Keep your legs comfortable apart and let your feet and knees relax completely, toes facing to the sides.

- Place your arms alongside, yet a little spread apart from your body. Leave your palms open, facing upward.

- Take your attention to different body parts one by one side; slowly relax your entire body.

- After some time, about 10 to 20 minutes when you feel fully relaxed, keeping your eyes closed; slowly roll onto your right side. Lie in that position for a minute or so.

\section{Advantages}

- Calm the soul and restore cells.

- Minimizes maternal stress.

\section{Yoga Nidra (Yogic Sleep)}

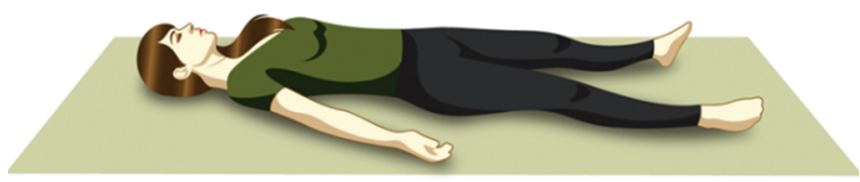

\section{Steps}

- Lie down straight as seen in the image. Shut your eyes and calm down. Inhale slowly and relaxed breaths.

- Gently focus your attention to your right extremity, concentrate for a few seconds, and meantime relax your foot. Gradually concentrate to the right extremity. Become conscious of your whole right lower extremity.

- Repeat this process for the left lower extremity.

- Concentrate to all parts of the body: private area, abdomen, navel region, and chest.

- Focus to the right shoulder, right arm, palms, and fingers. Redo this on the left shoulder, left arm, throat, face, and at last with the top of the head.

- Breathe deeply and note the sensations in your body. Calm in this state for a few minutes. 
- Gradually aware about your body and surroundings, twist your body to right side and continue lying down for a few more minutes. Rotate to the right side causes the breathe flow through the left nostril which helps to cool the body.

- Relax, then gradually sit up, and when you feel convenient, slowly, and gradually open your eyes.

\section{Advantages}

- Minimizes stress related to maternal.

- Aids in regulating BP.

- Deeply loosen up cells in the body.

\section{Pranayamas or Breathing Exercises DURING Pregnancy}

Pranayamas help to release negative emotions, such as anger and frustration. Keeps the mind calm and relaxed.

\section{Bhramari Pranayama (Bee Breath)}

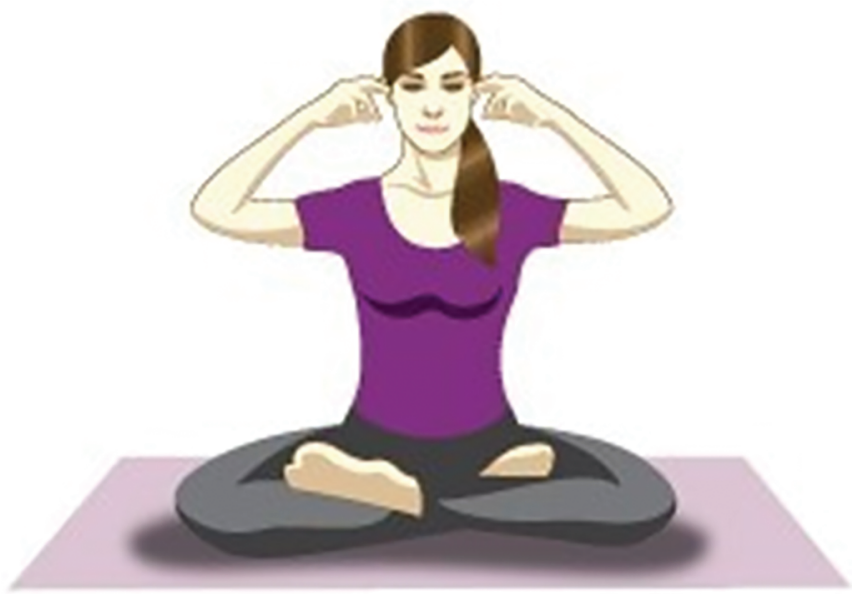

\section{Procedure}

- Position yourself in any other sitting asana.

- Shut your eyes and breathe deeply.

- Now shut your ears or flaps with your thumbs.

- Place your index finger just above eyebrows and the rest of your fingers over your eyes middle fingers.

- Give tender pressure to one side of your nose.

- Focus your mind in-between your eyebrows.

- Close your mouth; exhale slowly through your nose with Om sound.

- Repeat this process for five times. Important thing is that while doing this pranayama considering that you are being connected to all the positive energies of the universe.

\section{Nadi Shodhan Pranayama (Alternate Nostril Breathing} Technique)

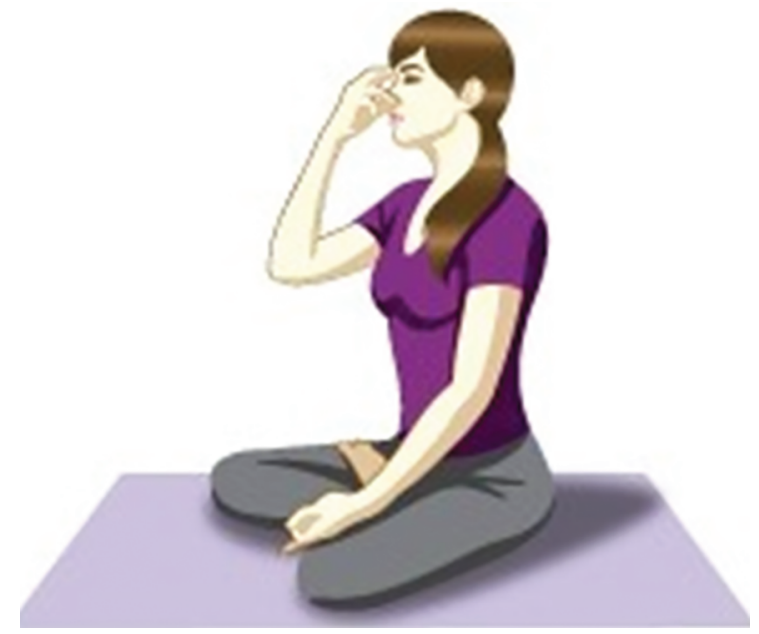

Steps

- Sit conveniently with your spine straight and shoulders relaxed

- Keep your left hand on the left knee, and palms open to the sky in Chin Mudra (thumb and index finger gently touching at the tips).

- Keep the tip of the index finger and middle finger of the right hand in-between the eyebrows, the ring finger and little finger on the left nostril, and the thumb on the right nostril. We will use the ring finger and little finger to open or close the left nostril and thumb for the right nostril.

- Give gentle pressure in thumb down on the right nostril and exhale gently through the left nostril.

- Now inhale from the left nostril and then press the left nostril gently with the ring finger and little finger. Removing the right thumb from the right nostril, breathe out from the right.

- Inhale from the right nostril and exhale from the left. You have now completed one round of this asana. Continue inhaling and exhaling from alternate nostrils.

- Complete nine such rounds by consequently breathing through both the nostrils. After every exhalation, remember to breathe in from the same nostril from which you exhaled. Keep your eyes closed throughout and continue taking long, deep, smooth breaths without any force or effort.

\section{Advantages}

- Calms and relaxes the mind.

- Maintains body temperature.

- Enhances oxygen supply which helps with the baby's growth.

- After practising these yoga moves and pranayamas, follow-up with a session of meditation. It will help you relax deeply.

\section{Yoga Precautions for an Expectant Mother}

- It helps regulate blood pressure.

- Relieves headaches.

- During the third trimester of pregnancy, avoid yoga asanas which tense the abdomen. 
- Throughout the first trimester of pregnancy, doing standing yoga poses. It aids in strengthening the legs and enhances circulation. It can even reduce leg cramps.

- During the second and the third trimesters, reduce the time spent holding asanas to prevent fatigue. Concentrate on doing breathing exercises and meditation.

- Do not practice yoga from the 10th to the 14th week of pregnancy since these are critical time.

- Avoid performing inversion poses.

- Perform as much as you can. ${ }^{10}$

\section{Yoga Posture to be Excluded during PregnanCY ${ }^{11}$}

- Naukasana (boat pose).

- Chakrasana (wheel pose).

- Ardha Matsyendrasana (sitting half spinal twist).

- Bhujangasana (cobra pose).

- Viparita Shalabhasana (superman pose).

- Halasana (plow pose). ${ }^{10}$

\section{Conclusion}

Yogic practice controls guides and strengthens the immune system, the miraculous birth process, healing and recovering from labor.

\section{References}

1. Beddoe $A E$, Lee $K A$. Mind-body interventions during pregnancy. J Obstet Gynecol Neonatal Nurs 2008;;37(no. 2):165-175. DOI: 10.1111/j.1552-6909.2008.00218.x.

2. Sandman CA, Davis EP, Buss C, Glynn LM. Prenatal programming of human neurological function. Int J Pept 2011;2011:837596. DOI: 10.1155/2011/837596.

3. Grizenko N, Fortier M-E, Zadorozny C, et al. Maternal stress during pregnancy, ADHD symptomatology in children, and genotype: gene-environment interaction. J Can Acad Child Adolesc Psychiatry 2012;21(no. 1):9-15.

4. Buss C, Davis EP, Hobel CJ, Sandman CA. Maternal pregnancy-specific anxiety is associated with child executive function at 69 years age. Stress 2011;14(6):665-676. DOI: 10.3109/10253890.2011.623250.

5. https://www.artofliving.org/in-en/yoga/yoga-for-women/yoga-andpregnancy.

6. https://parenting.firstcry.com/articles/yoga-pregnant-womenpostures-advantages-tips/.

7. Patel N. Yoga and Rehabilitation. 1st ed., Jaypee Publishers; 2008. pp. 222-223.

8. https://www.tommys.org/pregnancy-information/im-pregnant/ exercise-pregnancy/what-kind-exercises-can-i-do/yoga-pregnancy.

9. https://www.yogajournal.com/practice/yoga-for-moms-to-be.

10. Whitney ERD, Yoga Poses to Avoid During Pregnancy with Modifications. 17 Apr. 2018.

11. https://www.whitneyerd.com/2018/04/yoga-poses-to-avoid-duringpregnancy-with-modifications.html. 